\title{
Voltage Mode Astable Multivibrator Using Single CDBA
}

\author{
Rajeshwari Pandey, ${ }^{1}$ Neeta Pandey, ${ }^{1}$ Sajal K. Paul, ${ }^{2}$ \\ Kashish Anand, ${ }^{1}$ and Kranti Ghosh Gautam ${ }^{1}$ \\ ${ }^{1}$ Department of Electronics and Communication Engineering, Delhi Technological University, Bawana Road, \\ Delhi 110042, India \\ ${ }^{2}$ Department of Electronics Engineering, Indian School of Mines, Dhanbad, Jharkhand 826004, India
}

Correspondence should be addressed to Neeta Pandey; n66pandey@rediffmail.com

Received 21 January 2013; Accepted 14 February 2013

Academic Editors: H. A. Alzaher and H.-C. Chien

Copyright (C) 2013 Rajeshwari Pandey et al. This is an open access article distributed under the Creative Commons Attribution License, which permits unrestricted use, distribution, and reproduction in any medium, provided the original work is properly cited.

This paper aims at presenting three voltage mode square wave generator circuits using single current differencing buffered amplifier (CDBA), a recently proposed mixed mode building block. The first proposed circuit produces a variable frequency output having fixed duty cycle, whereas the rest of the circuits have variable duty cycle. One of the circuits uses passive element adjustment to control the duty cycle, whereas electronic control is used in the other circuit. The workability of the proposed circuits is confirmed through SPICE simulations and experimental work.

\section{Introduction}

It is well known that inherent wide bandwidth which is virtually independent of closed loop gain, greater linearity and large dynamic range [1] are the key performance features of current mode technique. The CDBA is one such active element which exploits these advantages. In addition, it is free from parasitic capacitances [2] and, hence, is appropriate for high frequency operation. It provides further flexibility to the designers, enabling a variety of circuit designs, as it can operate in both current and voltage modes [3].

The square wave generator finds extensive applications in communication systems, control systems, instrumentation, and signal processing. The literature review reveals that several triangular/square wave generators using various analog building blocks have already been presented [4-11]. All these designs use a current source to alternately charge and discharge a capacitor followed by a Schmitt trigger to generate triangular/square wave. Conventional voltage-mode square wave generators [4] employ an op-amp working in the nonlinear region to produce a square wave signal. These voltage-mode generators, however, have a limitation on their maximum frequency due to lower slew rate and constant gain-bandwidth product of the op-amps.

Triangular/square wave generators presented in [5-11] use current mode building-blocks-based circuits. The study of these circuits reveals that

(i) references $[5,6,10]$ employ more than one active element;

(ii) references [6-10] use four or more passive elements;

(iii) references [6-11] lack electronic controllability of duty cycle of output.

The aim of this paper is to present three square wave generator circuits using single CDBA and three to five passive elements. In Section 2, the function of a CDBA is introduced followed by the description of proposed circuits with analytical formulation for the frequency of oscillation. Section 3 explores the effect of nonidealities of CDBA on the proposed circuits. PSPICE simulation results and experimental results are presented in Section 4 which are in confirmation with the theoretical propositions. Section 5 concludes the paper. 


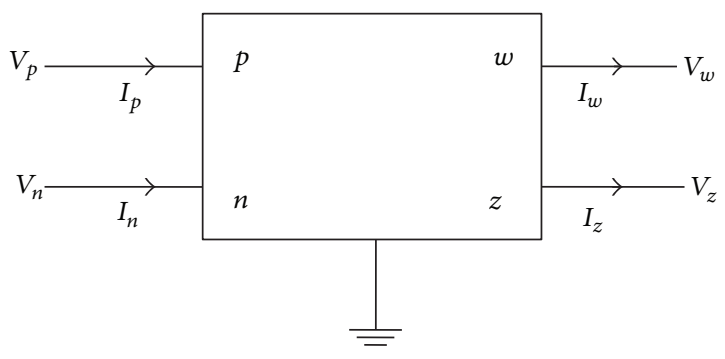

FIGURE 1: Block diagrammatic representation of CDBA.

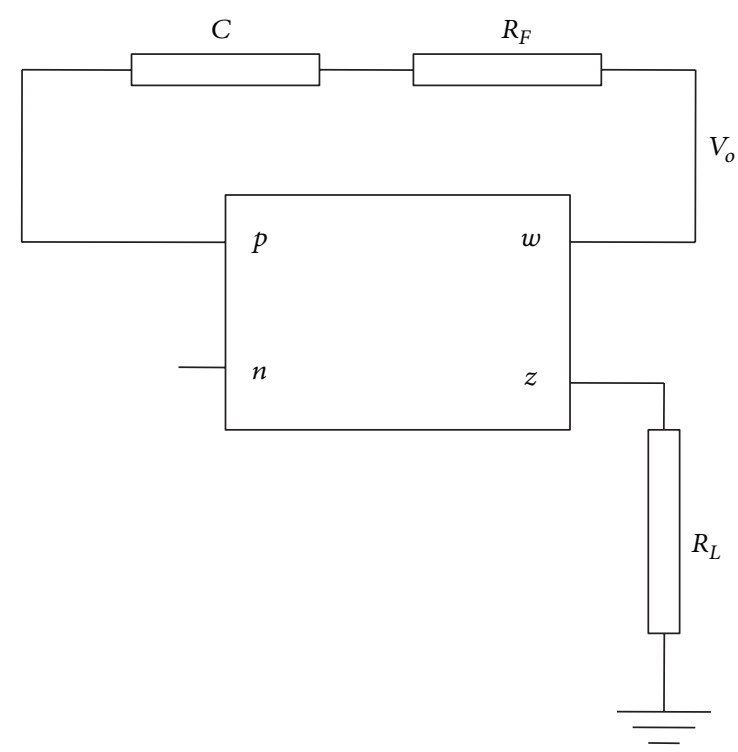

Figure 2: Astable multivibrator with fixed 50\% duty cycle.

\section{Circuit Descriptions}

The circuit symbol of CDBA is shown in Figure 1. The port characteristics are given as follows

$$
\left[\begin{array}{c}
I_{z} \\
V_{w} \\
V_{p} \\
V_{n}
\end{array}\right]=\left[\begin{array}{cccc}
0 & 0 & 1 & -1 \\
1 & 0 & 0 & 0 \\
0 & 0 & 0 & 0 \\
0 & 0 & 0 & 0
\end{array}\right]\left[\begin{array}{c}
V_{z} \\
I_{w} \\
I_{p} \\
I_{n}
\end{array}\right] .
$$

2.1. Circuit I. The circuit for the astable multivibrator is shown in Figure 2. It uses a single CDBA block, two resistors and a capacitor. The resistor $R_{F}$ and capacitor $C$ form a positive feedback loop. The load resistance $R_{L}$ connected to the $z$ terminal is large enough to drive the device output $V_{0}$ into one of the two saturation levels $V_{\text {sat }}^{+}$or $V_{\text {sat. }}^{-}$. This results in charging of the capacitor present in the feedback loop. When the voltage across the capacitor reaches a value at which the current through $R_{L}$ is not large enough to maintain the output voltage at $V_{\text {sat }}^{+}$the output switches to $V_{\text {sat }}^{-}$and the capacitor starts charging in the opposite direction as shown in Figure 3.

Routine analysis suggests that the output will switch between its maximum $\left(V_{\text {sat }}^{+}\right)$and minimum $\left(V_{\text {sat }}^{-}\right)$value when

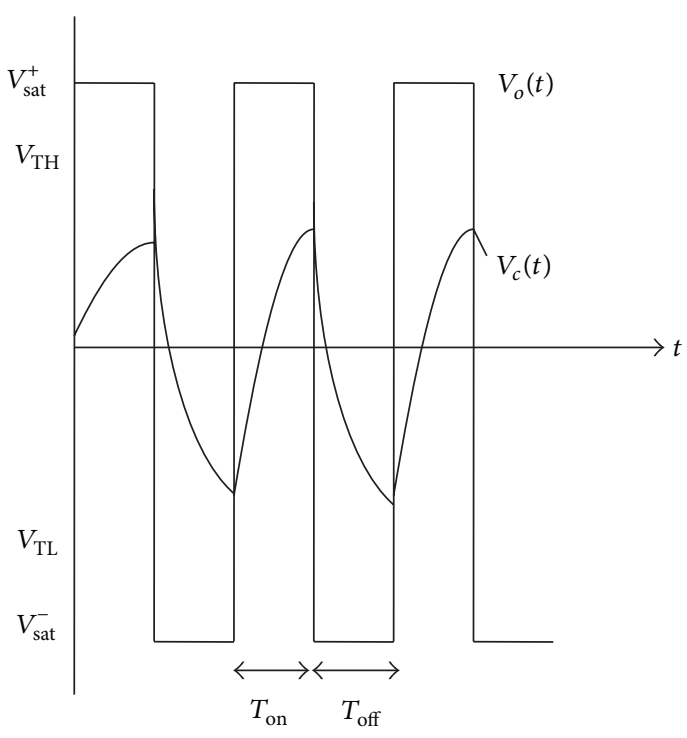

Figure 3: Output of the astable multivibrator of Figure 2.

the capacitor voltage reaches the following two threshold voltages:

$$
\begin{gathered}
V_{\mathrm{TL}}=\frac{\left(R_{L}-R_{F}\right)}{R_{L}} V_{\text {sat }}^{-}, \\
V_{\mathrm{TH}}=\frac{\left(R_{L}-R_{F}\right)}{R_{L}} V_{\text {sat }}^{+} .
\end{gathered}
$$

The time required to charge $\left(T_{\text {on }}\right)$ the capacitor from a value of $V_{\mathrm{TL}}$ to $V_{\mathrm{TH}}$ is given by

$$
T_{\text {on }}=R_{F} C \ln \left[\frac{2 R_{L}}{R_{F}}-1\right] .
$$

Similarly, time taken for discharging ( $\left.T_{\text {off }}\right)$ from $V_{\mathrm{TH}}$ to $V_{\mathrm{TL}}$ is

$$
T_{\text {off }}=R_{F} C \ln \left[\frac{2 R_{L}}{R_{F}}-1\right] .
$$

From (4) and (5), it is clear that $R_{L}>R_{F}$.

Thus, the output of the multivibrator is a symmetric square wave having amplitudes of $V_{\text {sat }}^{+}$and $V_{\text {sat }}^{-}$, at a frequency $\left(f_{o}\right)$ given by

$$
f_{o}=\frac{1}{T_{\text {on }}+T_{\text {off }}}=\frac{1}{2 R_{F} C \ln \left[2 R_{L} / R_{F}-1\right]} .
$$

Though the circuit is simple and output frequency can be controlled through passive components, however, the on and off duty cycles of the output are fixed at $50 \%$.

2.2. Circuit II. The circuit of Figure 2 can be modified for variable duty cycle output by replacing the resistor $R_{F}$ with two diodes and two resistors and is depicted in Figure 4. It is 


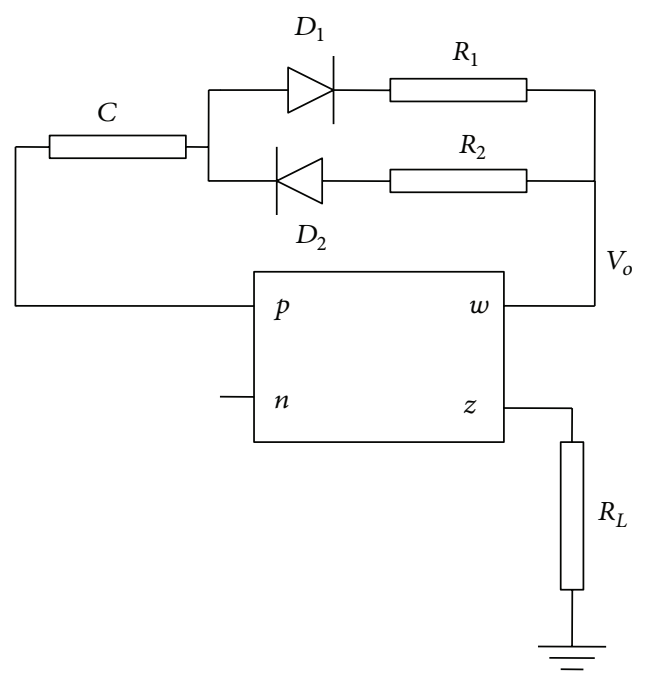

FIGURE 4: Astable multivibrator having resistor-controlled duty cycle.

based on the scheme discussed in [11] and has been adapted for implementation with CDBA.

Neglecting the drop across the diodes, the parameters $V_{\mathrm{TH}}, V_{\mathrm{TL}}, T_{\text {on }}$ and $T_{\text {off }}$ may be obtained as

$$
\begin{gathered}
V_{\mathrm{TL}}=\frac{\left(R_{L}-R_{1}\right)}{R_{L}} V_{\text {sat }}^{-}, \\
V_{\mathrm{TH}}=\frac{\left(R_{L}-R_{2}\right)}{R_{L}} V_{\text {sat }}^{+}, \\
T_{\text {on }}=R_{2} C \ln \left(\frac{2 R_{L}-R_{1}}{R_{2}}\right), \\
T_{\text {off }}=R_{1} C \ln \left(\frac{2 R_{L}-R_{2}}{R_{1}}\right) .
\end{gathered}
$$

From (9) and (10), the frequency $\left(f_{o}\right)$ of modified square wave generator can be written as

$$
\begin{array}{r}
f_{o}=\frac{1}{R_{2} C \ln \left[\left(2 R_{L}-R_{1}\right) / R_{2}\right]+R_{1} C \ln \left[\left(2 R_{L}-R_{2}\right) / R_{1}\right]}, \\
\text { where } R_{L}>R_{1}, R_{2} .
\end{array}
$$

It can be seen clearly that $T_{\text {on }}$ and $T_{\text {off }}$ can be controlled by changing $R_{2}$ and $R_{1}$.

2.3. Circuit III. The circuit shown in Figure 5 is another astable multivibrator. In this circuit, the duty cycle can be electronically controlled by the application of an external DC source $V_{\mathrm{dc}}$.

Routine analysis for this circuit gives the $V_{\mathrm{TH}}$ and $V_{\mathrm{TL}}$ as

$$
\begin{gathered}
V_{\mathrm{TH}}=V_{\text {sat }}^{+}\left(1-\frac{R_{F}}{R_{L}}\right)+V_{\mathrm{dc}} \frac{R_{F}}{R_{S}}, \\
V_{\mathrm{TL}}=V_{\text {sat }}^{-}\left(1-\frac{R_{F}}{R_{L}}\right)+V_{\mathrm{dc}} \frac{R_{F}}{R_{S}} .
\end{gathered}
$$

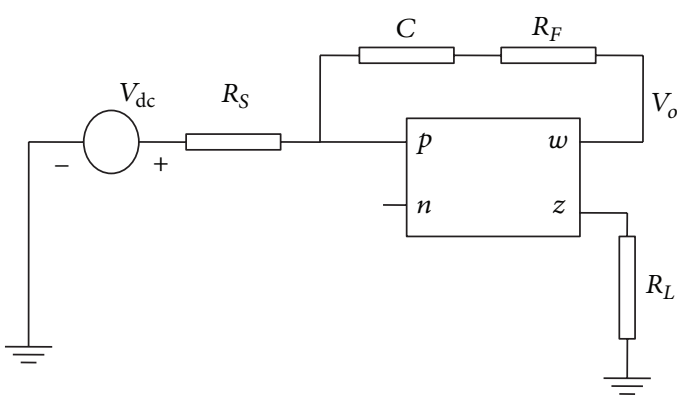

FIGURE 5: Astable multivibrator having electronically controlled duty cycle.

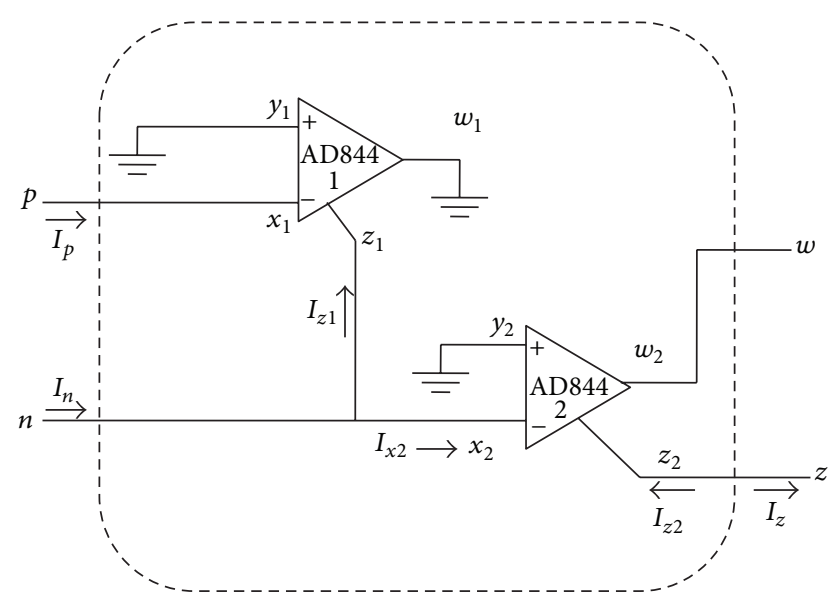

Figure 6: CDBA constructed with AD844.

These values of $V_{\mathrm{TH}}$ and $V_{\mathrm{TL}}$ result in $T_{\mathrm{on}}$ and $T_{\mathrm{off}}$ as follows:

$$
\begin{aligned}
& T_{\text {on }}=R C \ln \frac{\left[2 R_{L} / R_{F}-1\right]-R_{L} V_{\mathrm{dc}} / V_{\mathrm{sat}}^{+} R_{S}}{\left[1-V_{\mathrm{dc}} R_{L} / V_{\mathrm{sat}}^{+} R_{S}\right]}, \\
& T_{\mathrm{off}}=R C \ln \frac{\left[2 R_{L} / R_{F}-1\right]-V_{\mathrm{dc}} R_{L} / V_{\mathrm{sat}}^{-} R_{S}}{\left[1-V_{\mathrm{dc}} R_{L} / V_{\mathrm{sat}}^{-} R_{S}\right]} .
\end{aligned}
$$

These results show that the on and off duty cycles of the output can be controlled with the help of externally applied voltage $V_{\mathrm{dc}}$.

\section{Realizing a CDBA and Nonideality Analysis}

In the analysis so far, ideal characteristics of the CDBA were considered. However, in this section, the effect of the parameters of a practical model of the CDBA is investigated. For the proposed circuits, the CDBA was realized using current feedback operational amplifier (CFOA) ICAD844 as shown in Figure 6 [12]. The equivalent circuit of Figure 6, which uses practical model of AD844 [13], is shown in Figure 7. Herein, the CFOAs have been replaced with current conveyors having finite input resistances $\left(R_{x}\right)$ and finite resistance at its $z$ terminal $\left(R_{z}\right)$. Ideally, the input resistance at the $x$ terminal is zero and is infinite at the $z$ terminal. For the AD844 CFOA, the input resistances $R_{x}=50 \Omega$ and $R_{z}=3 \mathrm{M} \Omega$ [13]. This 


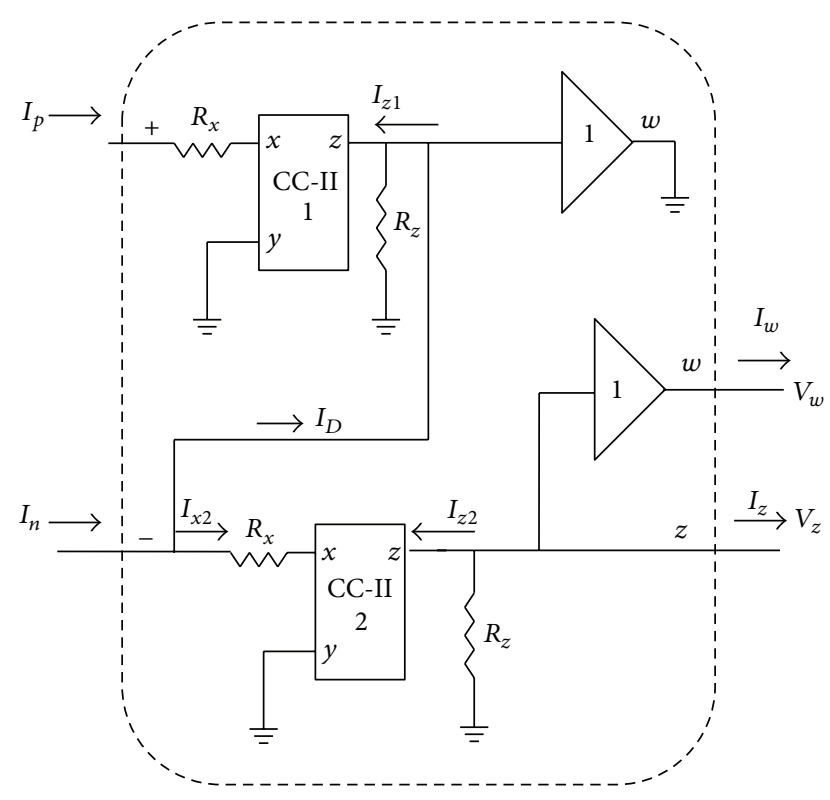

Figure 7: Equivalent circuit of CDBA constructed with AD844.

circuit is used for exploring the nonideal behavior of all the three proposed circuits.

From Figure 7, various currents can be calculated as follows:

$$
\begin{gathered}
I_{z 1}=I_{p}, \\
I_{D}=I_{z 1}\left(\frac{R_{z}}{R_{x}+R_{z}}\right), \\
I_{x 2}=I_{n}-I_{D}, \\
I_{z 2}=I_{x 2} .
\end{gathered}
$$

Ideally, $I_{D}$ should be equal to $I_{z 1}$, which can be approximated only if $R_{z} \gg R_{x}$, which is true for AD844. Also, the approximation that the input terminals are virtually grounded will be true only if the external resistance at the input terminal of the CDBA is much larger than $R_{x}$. If these two conditions are satisfied, the CDBA constructed with AD844 closely approximates an ideal CDBA.

Taking into account the afore mentioned approximations, from (15), $I_{z}$, the current from $z$ terminal, can be calculated as

$$
I_{z}=\left(I_{p}-I_{n}\right)
$$

And the output voltage $V_{w}$ is given as

$$
V_{w}=V_{z}
$$

If the equivalent circuit of CDBA constructed with AD844 is used in Figure 2 (circuit I), then the threshold limits of the output get modified to

$$
\begin{gathered}
V_{\mathrm{TL}}=\frac{\left(R_{L} / / R_{z}\right)-\left(R_{F}+R_{x}\right)}{R_{L} / / R_{z}} V_{\text {sat }}^{-}, \\
V_{\mathrm{TH}}=\frac{\left(R_{L} / / R_{z}\right)-\left(R_{F}+R_{x}\right)}{R_{L} / / R_{z}} V_{\text {sat }}^{+} .
\end{gathered}
$$

Since $R_{L} \ll R_{z}$ and $R_{F} \gg R_{x}$, (18) and (19) reduce to (2) and (3), respectively.

The threshold limits of the output of Figure 4 (circuit II), on using the equivalent CDBA model, get modified to

$$
\begin{gathered}
V_{\mathrm{TL}}=\frac{\left(R_{L} / / R_{z}\right)-\left(R_{1}+R_{x}+R_{D 1}\right)}{R_{L} / / R_{z}} V_{\text {sat }}^{-}, \\
V_{\mathrm{TH}}=\frac{\left(R_{L} / / R_{z}\right)-\left(R_{2}+R_{x}+R_{D 2}\right)}{R_{L} / / R_{z}} V_{\text {sat }}^{+}
\end{gathered}
$$

where $R_{D 1}$ and $R_{D 2}$ are forward resistances of the diodes $D_{1}$ and $D_{2}$, respectively, and are of very small order as compared to $R_{1}$ and $R_{2}$. Also, since $R_{x} \ll R_{F}$ and $R_{z} \gg R_{L},(20)$ and (21) can be approximated to (7) and (8), respectively.

Similarly, for the astable circuit shown in Figure 5 (circuit III), the modified threshold limits of the output can be expressed as

$$
\begin{aligned}
& V_{\mathrm{TH}}=V_{\text {sat }}^{+}\left(1-\frac{R_{F}+R_{x}}{R_{L} / / R_{z}}\right)+V_{\mathrm{dc}} \frac{R_{F}+R_{x}}{R_{S}+R_{x}}, \\
& V_{\mathrm{TL}}=V_{\text {sat }}^{-}\left(1-\frac{R_{F}+R_{x}}{R_{L} / / R_{z}}\right)+V_{\mathrm{dc}} \frac{R_{F}+R_{x}}{R_{S}+R_{x}} .
\end{aligned}
$$

The external resistance at the input of the CDBA should be much larger than $R_{x}$ so that the feedback current can be absorbed into the input terminals. Since $R_{L}, R_{F}, R_{S}$, and $R_{z} \gg$ $R_{x},(22)$ and (23) reduce to (12) and (13), respectively.

\section{Simulation and Experimental Results}

To validate the theoretical predictions, the proposed astable multivibrator circuits have been simulated using PSPICE macromodel of current feedback operational amplifier (CFOA) AD 844 and experimented using commercially available AD 844AN. The CDBA realization using CFOA $\mathrm{AD} 844$ is shown in Figure 6. Simulations were carried out to investigate the maximum and minimum frequencies that proposed circuit configuration of Figure 2 (circuit I) can offer before the output signal gets distorted. Supply voltage of $\pm 10 \mathrm{~V}$ was used for simulations. Figure 8 shows the output of the proposed circuit for minimum and maximum frequencies which can be achieved, with frequency deviation not more than $5 \%$. The simulated square wave output obtained with component values as $R_{F}=20 \mathrm{~K} \Omega, R_{L}=50 \mathrm{~K} \Omega$ and $C=10 \mu \mathrm{F}$ is shown in Figure 8(a). This is the minimum frequency that can be obtained from the circuit and its value is observed to be $2 \mathrm{~Hz}$ as against the theoretically computed value of $1.9 \mathrm{~Hz}$. Similarly output for maximum achievable frequency is shown in Figure 8(b). Component values used are $R_{F}=1 \mathrm{~K} \Omega, R_{L}=$ $40 \mathrm{~K} \Omega$ and $C=100 \mathrm{pF}$. The simulated frequency is noted to be $1.2 \mathrm{MHz}$ showing a minor deviation from the theoretically calculated value of $1.14 \mathrm{MHz}$.

To test the tunability of this circuit, the variation in frequency, against the passive components $C, R_{L}$, and $R_{F}$, was observed. The variation of frequency against the capacitor $C$ is presented in Figure 9(a), whereas Figures 9(b) and 9(c) plot the variations against the resistors $R_{L}$ and $R_{F}$, respectively. 


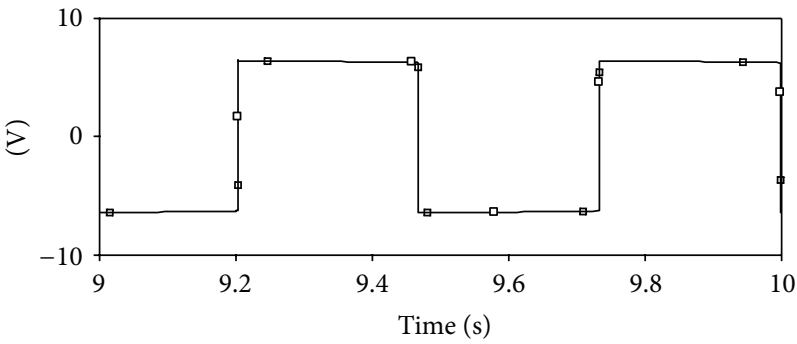

$\square V_{0}$

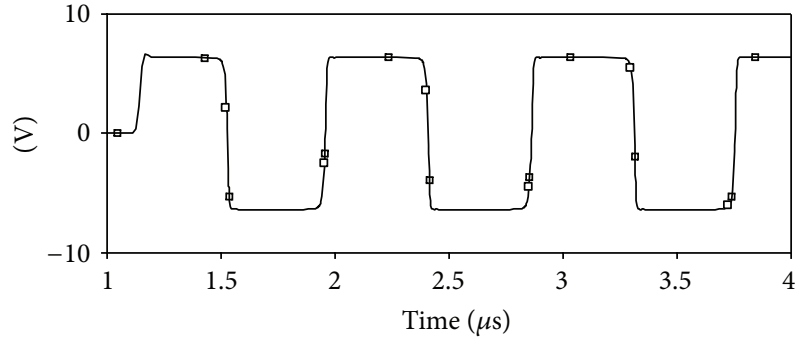

$V_{0}$

(a) Minimum frequency output

(b) Maximum frequency output

FIgURE 8: Output of circuit I.

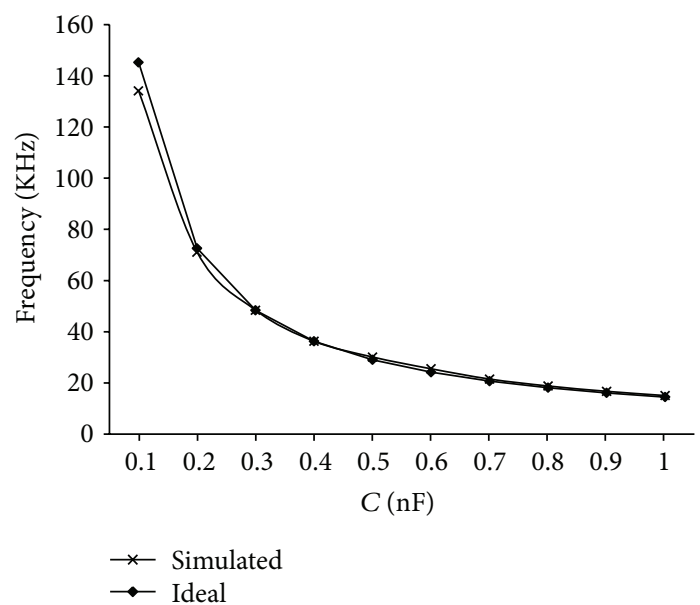

(a)

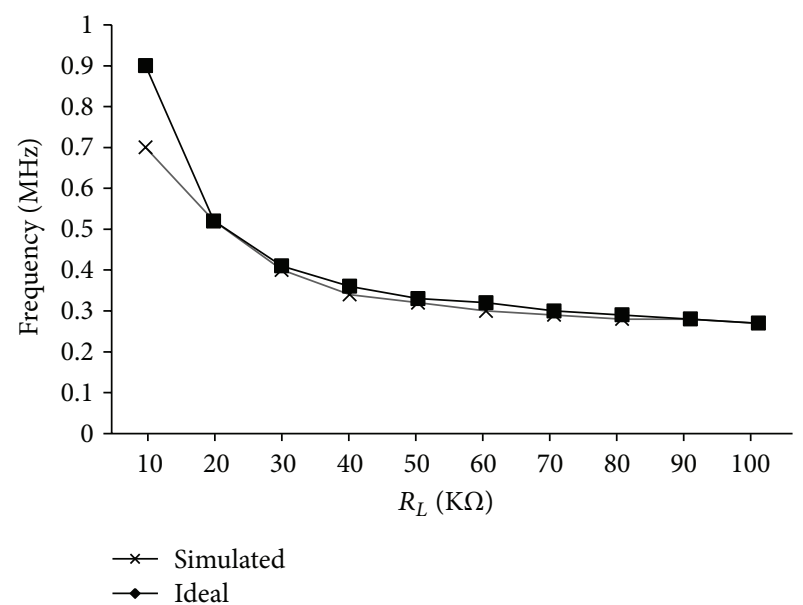

(b)

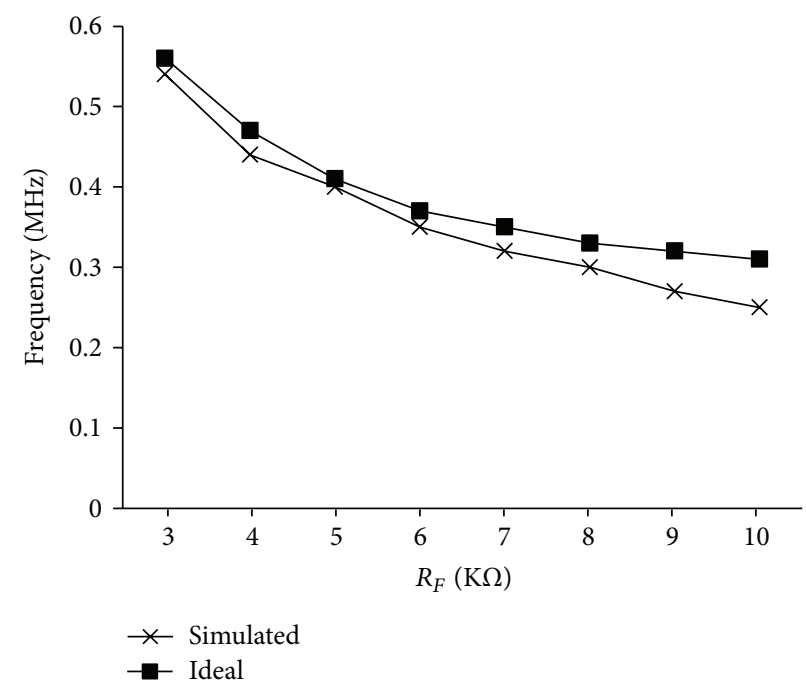

(c)

FIGURE 9: (a) Variation of calculated and simulated values of frequency with $C$ for $R_{F}=15 \mathrm{~K} \Omega$ and $R_{L}=40 \mathrm{~K} \Omega$. (b) Variation of calculated and simulated values of frequency with $R_{L}$ for $C=100 \mathrm{pF}$ and $R_{F}=5 \mathrm{~K} \Omega$. (c) Variation of calculated and simulated values of frequency with $R_{F}$ for $C=100 \mathrm{pF}$ and $R_{L}=30 \mathrm{~K} \Omega$. 


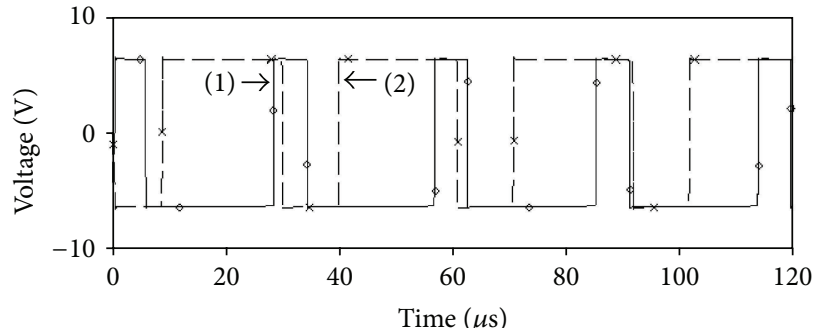

(a)

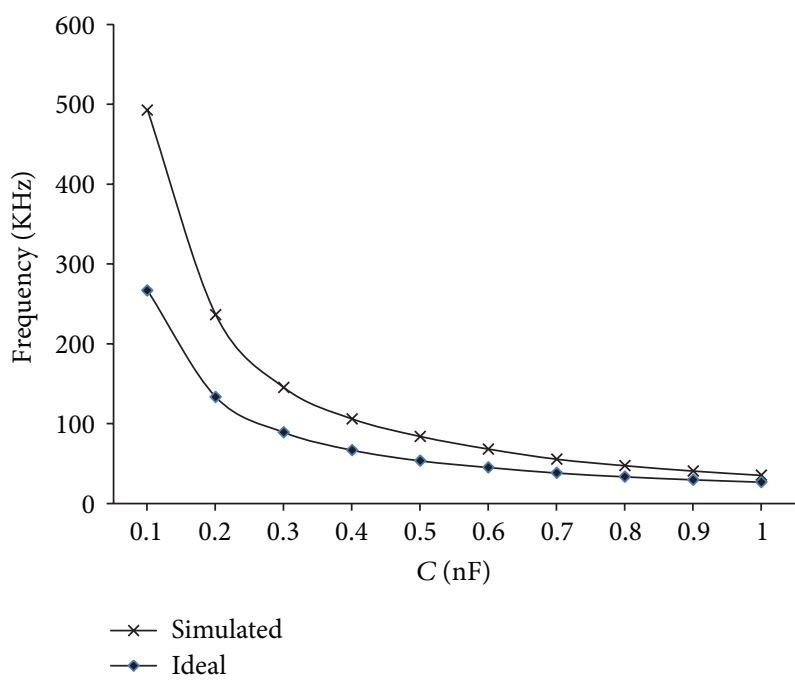

(b)

FIGURE 10: (a) Output of circuit II. (b) Variation of calculated and simulated values of frequency with $C$.

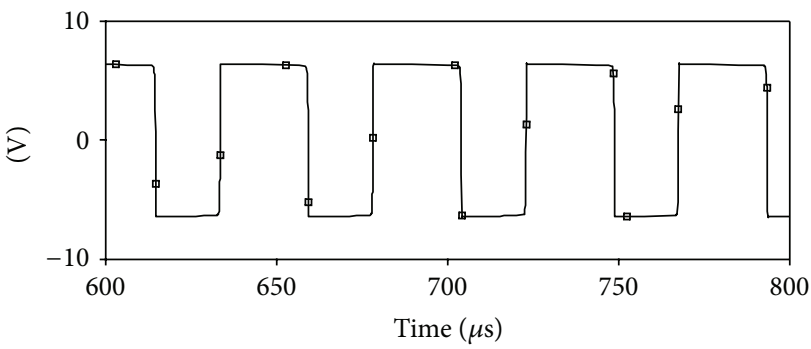

$\square V_{0}$

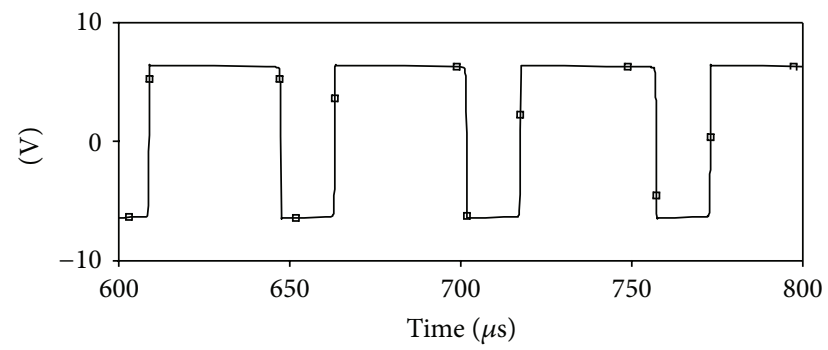

$\square V_{0}$

(a)

(b)

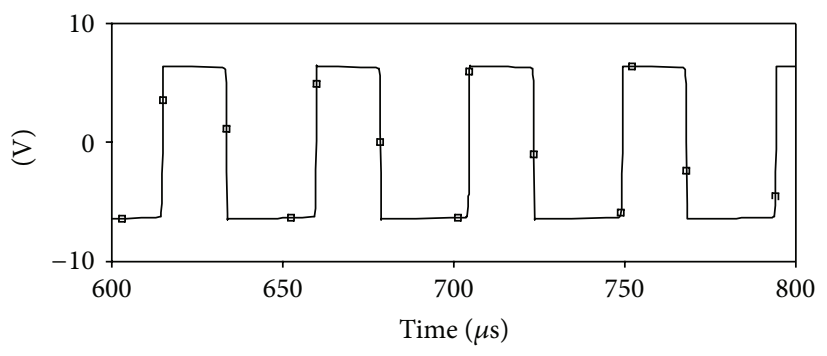

$V_{0}$

(c)

FIGURE 11: (a) Output of circuit III with $V_{\mathrm{dc}}=2 \mathrm{~V}$. (b) Output of circuit III with $V_{\mathrm{dc}}=5 \mathrm{~V}$. (c) Output of circuit III with $V_{\mathrm{dc}}=-2 \mathrm{~V}$.

The circuit configuration of Figure 4 (circuit II) was simulated to investigate the variation of duty cycle by changing the passive component values. A typical output of this circuit is given in Figure 10(a), wherein curve (1) is plotted for $R_{1}=$ $25 \mathrm{~K} \Omega$ and $R_{2}=2 \mathrm{~K} \Omega$ and curve (2) for $R_{1}=4 \mathrm{~K} \Omega$ and $R_{2}=15 \mathrm{~K} \Omega$. The values of $R_{L}$ and $C$ are kept as $40 \mathrm{~K} \Omega 1 \mathrm{nF}$, respectively, for both curves. It clearly shows that the on and off periods of the output are not fixed anymore. Figure 10(b) shows the plot of the calculated frequency and the simulated frequency of oscillation as a function of $C$ while keeping $R_{L}=40 \mathrm{~K} \Omega, R_{1}=20 \mathrm{~K} \Omega$, and $R_{2}=4 \mathrm{~K} \Omega$. The deviation in frequency arises since the voltage drop across the diodes and their forward resistance is not taken into account in calculations.

Figure 11 shows the output of circuit III for three different values of $V_{\mathrm{dc}}$, that is, $2 \mathrm{~V}, 5 \mathrm{~V}$, and $-2 \mathrm{~V}$, while rest of the 


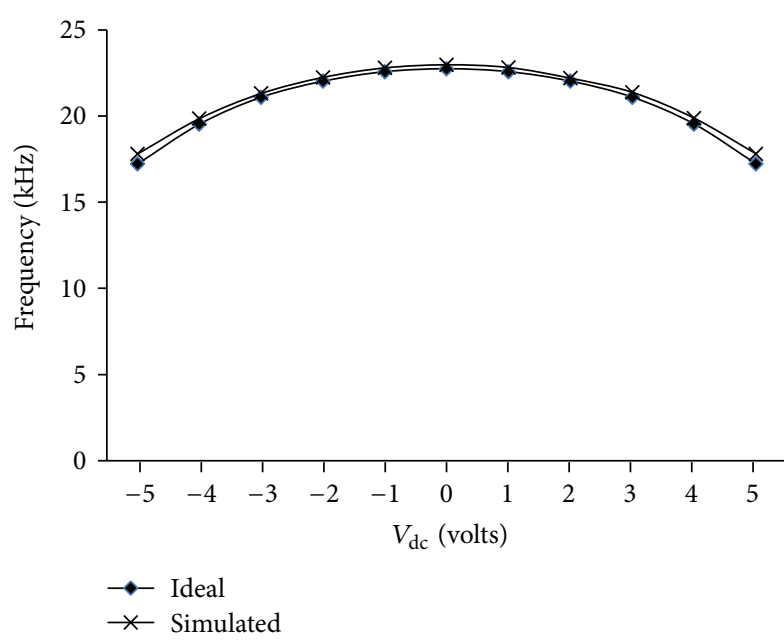

(a)

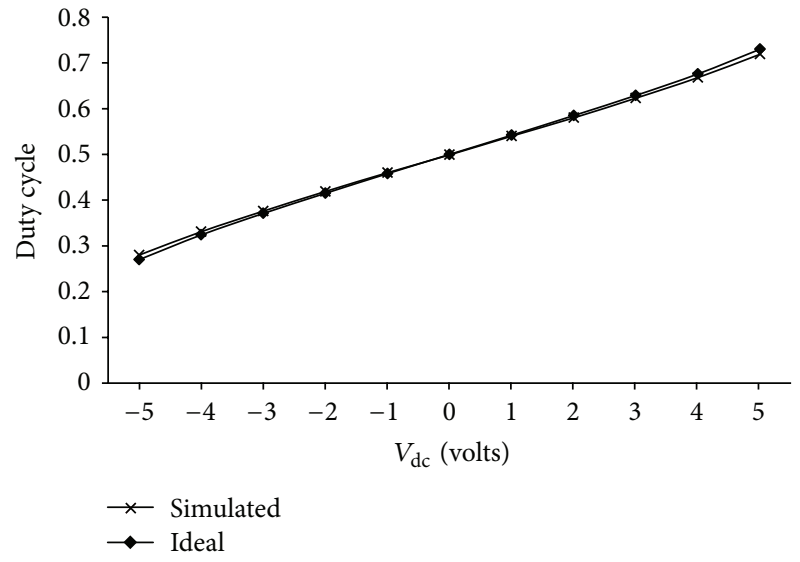

(b)

FIGURE 12: (a) Variation of calculated and simulated value of frequency with DC bias. (b) Variation of duty cycle with DC bias.

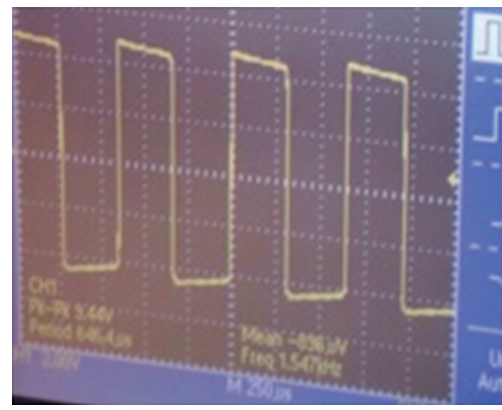

(a)

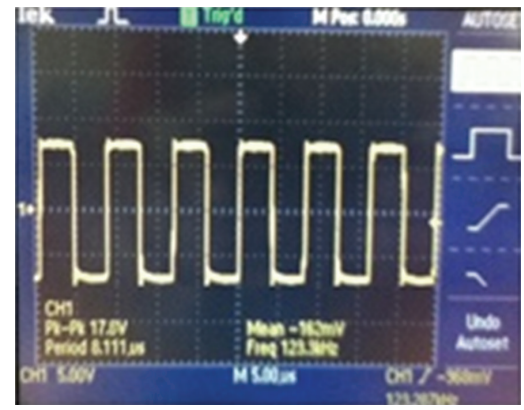

(b)

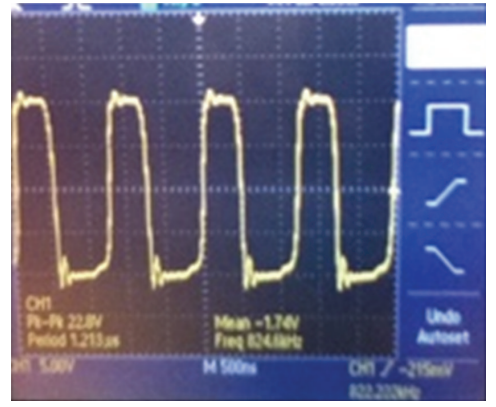

(c)

FIgURE 13: Experimental output obtained from circuit I.

component values are chosen as $R_{s}=40 \mathrm{~K}, R_{L}=40 \mathrm{~K}, R_{F}=$ $15 \mathrm{~K}$, and $C=1 \mathrm{nF}$. It is evident that the output frequency and duty cycle can be controlled using $V_{\mathrm{dc}}$.

Figure 12(a) shows the comparison between the calculated frequency and the simulated frequency of oscillations as a function of $V_{\mathrm{dc}}$, and Figure 12(b) shows variation of duty cycle with $V_{\mathrm{dc}}$, for component values of $R_{S}=40 \mathrm{~K}, R_{L}=40 \mathrm{~K}$, $R_{F}=15 \mathrm{~K}$, and $C=1 \mathrm{nF}$. It is observed that all the simulated results are found in close agreement with the theoretically formulated values.

The functionality of the proposed square wave generator circuits is verified experimentally as well. The commercial IC $\mathrm{AD} 844 \mathrm{AN}$ is used to implement a CDBA. Supply voltages used are $\pm 10 \mathrm{~V}$.

Figure 13 shows typical experimental results for circuit I for three different frequencies. Figure 13(a) is output for component values of $R_{L}=82 \mathrm{~K} \Omega, R_{F}=15 \mathrm{~K} \Omega$, and $C=$ $10 \mathrm{nF}$. The measured frequency of $1.547 \mathrm{KHz}$ closely matches the theoretical frequency of $1.46 \mathrm{KHz}$ as obtained from (6). Output for component values of $R_{L}=3.9 \mathrm{~K} \Omega, R_{F}=1 \mathrm{~K} \Omega$, and $C=2 \mathrm{nF}$ is shown in Figure 13(b), wherein the measured frequency of oscillation is $123.3 \mathrm{KHz}$ as against the computed value of $130 \mathrm{KHz}$. Another screen shot of oscilloscope is shown in Figure 13(c) for $R_{L}=40 \mathrm{~K} \Omega, R_{F}=1 \mathrm{~K} \Omega$, and $C=$ $100 \mathrm{pF}$. Observed output is having a frequency of oscillation as $824 \mathrm{KHz}$ and the corresponding calculated value is found to be $1.14 \mathrm{MHz}$. Experimental outputs for the circuit III are shown in Figure 14 for different values of $V_{\mathrm{dc}}$, which depicts the variation of duty cycle with $V_{\mathrm{dc}}$.

\section{Conclusion}

Three astable multivibrator circuits using single CDBA are proposed. The first circuit (circuit I) produces a variable frequency output with fixed duty cycle. The other two proposed circuits (circuit II and circuit III) provide output with variable duty cycle; for circuit II, duty cycle control is accomplished through passive component adjustment, and for circuit III, electronic control is used. Nonideality analysis is presented to explore the effect of the parameters of a practical model of the CDBA. The workability of the proposed circuits is demonstrated through SPICE simulations and experimental work. AD-844-based CDBA is used for simulation and 


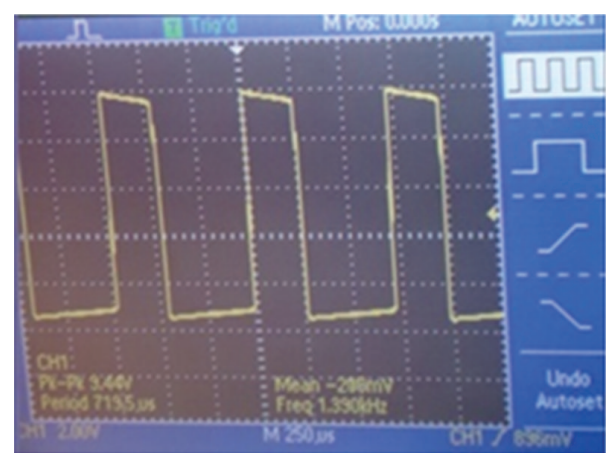

(a)

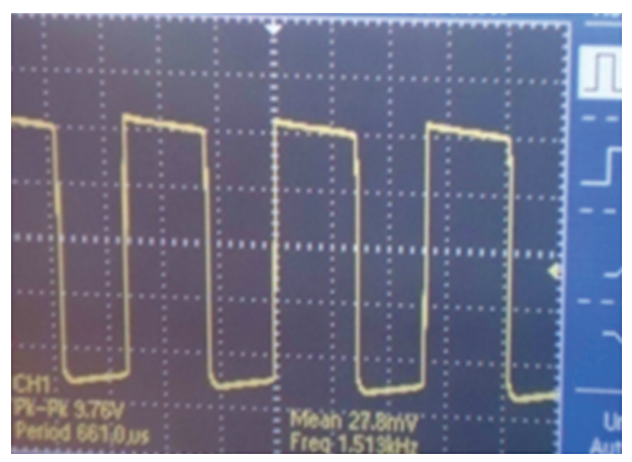

(b)

FIGURE 14: Experimental outputs for circuit III for (a) $V_{\mathrm{dc}}=-5 \mathrm{~V}$ and (b) $V_{\mathrm{dc}}=2 \mathrm{~V}$.

hardware circuit tests. Results are found to be in conformity with the proposed theory.

\section{References}

[1] C. Toumazou, F. J. Lidgey, and D. G. Haigh, Analogue IC Design: The Current Mode Approach, Peregrinus, London, UK, 1990.

[2] C. Acar and S. Ozoguz, "A new versatile building block: current differencing buffered amplifier suitable for analog signalprocessing filters," Microelectronics Journal, vol. 30, no. 2, pp. 157-160, 1999.

[3] C. Cakir and O. Cicekoglu, "Low-voltage high-performance CMOS current differencing buffered amplifier (CDBA)," in Proceedings of the 4th Ph.D. Research in Microelectronics and Electronics Conference (PRIME '08), pp. 37-40, June 2008.

[4] A. Sedra and K. C. Smith, Microelectronic Circuits, Oxford University Press, London, UK, 4th edition, 1998.

[5] W.-S. Chung, H. Kim, H. W. Cha, and H. J. Kim, "Triangular/square-wave generator with independently controllable frequency and amplitude," IEEE Transactions on Instrumentation and Measurement, vol. 54, no. 1, pp. 105-109, 2005.

[6] D. Pal, A. Srinivasulu, B. B. Pal, A. Demosthenous, and B. N. Das, "Current conveyor-based square/triangular waveform generators with improved linearity," IEEE Transactions on Instrumentation and Measurement, vol. 58, no. 7, pp. 2174-2180, 2009.

[7] B. Almashary and H. Alhokail, "Current-mode triangular wave generator using CCIIs," Microelectronics Journal, vol. 31, no. 4, pp. 239-243, 2000.
[8] M. T. Abuelma'atti and S. M. Al-Shahrani, "New CFOA-based triangular/square wave generator," International Journal of Electronics, vol. 84, no. 6, pp. 583-588, 1998.

[9] M. B. Amer and M. A. Ibbini, "Novel single-element- controlled CFOA-based square wave oscillator," in Proceedings of the IEEE Conference on Systems Signals and Devices, pp. 1-5, 2005.

[10] A. K. M. S. Haque, M. M. Hossain, W. A. Davis, H. T. Russell, and R. L. Carter, "Design of sinusoidal, triangular, and square wave generator using current feedback operational amplifier (CFOA)," in Proceedings of the IEEE Region 5 Conference, pp. 1-5, April 2008.

[11] C. L. Hou, H. C. Chien, and Y. K. Lo, "Square wave generators employing OTRAs," IEE Proceedings Circuits, Devices \& Systems, vol. 152, no. 6, pp. 718-722, 2005.

[12] S. Ozcan, A. Toker, C. Acar, H. Kuntman, and O. Cicekoglu, "Single resistance-controlled sinusoidal oscillators employing current differencing buffered amplifier," Microelectronics Journal, vol. 31, no. 3, pp. 169-174, 2000.

[13] AD844 Current Feedback Op-Amp Data Sheet, Analog Devices Inc., Norwood, Mass, USA, 1990. 

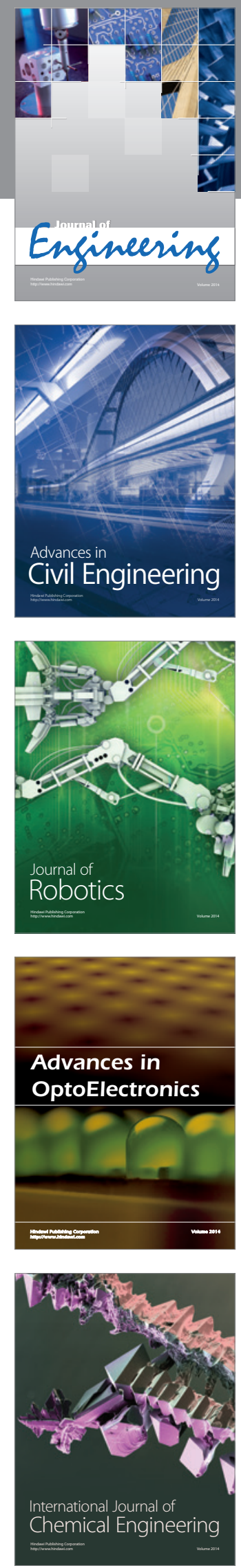

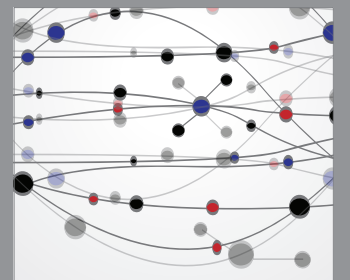

The Scientific World Journal
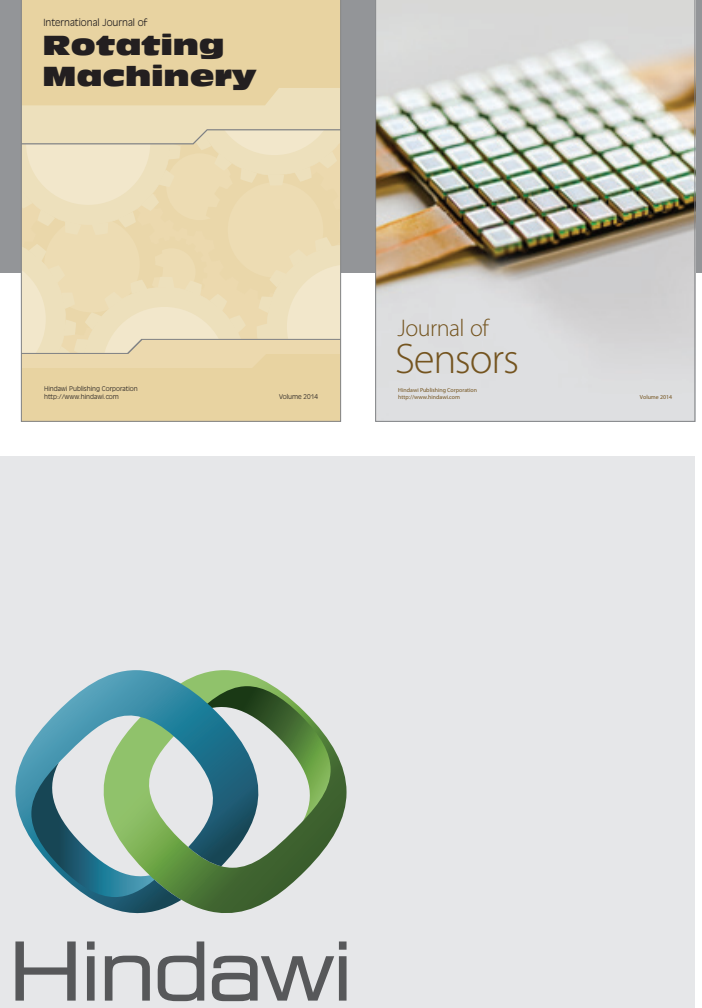

Submit your manuscripts at http://www.hindawi.com
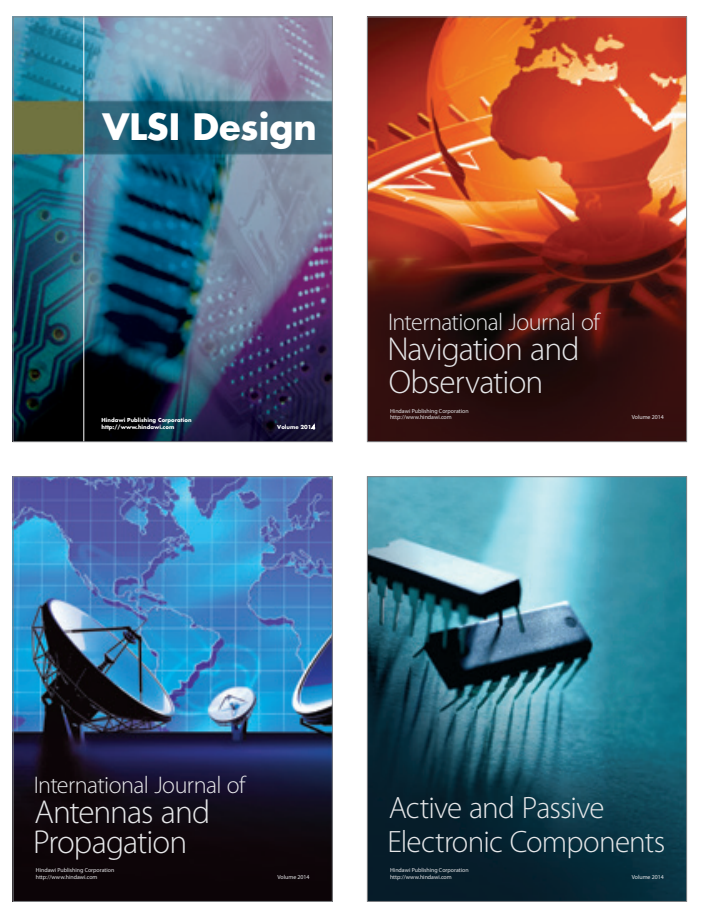
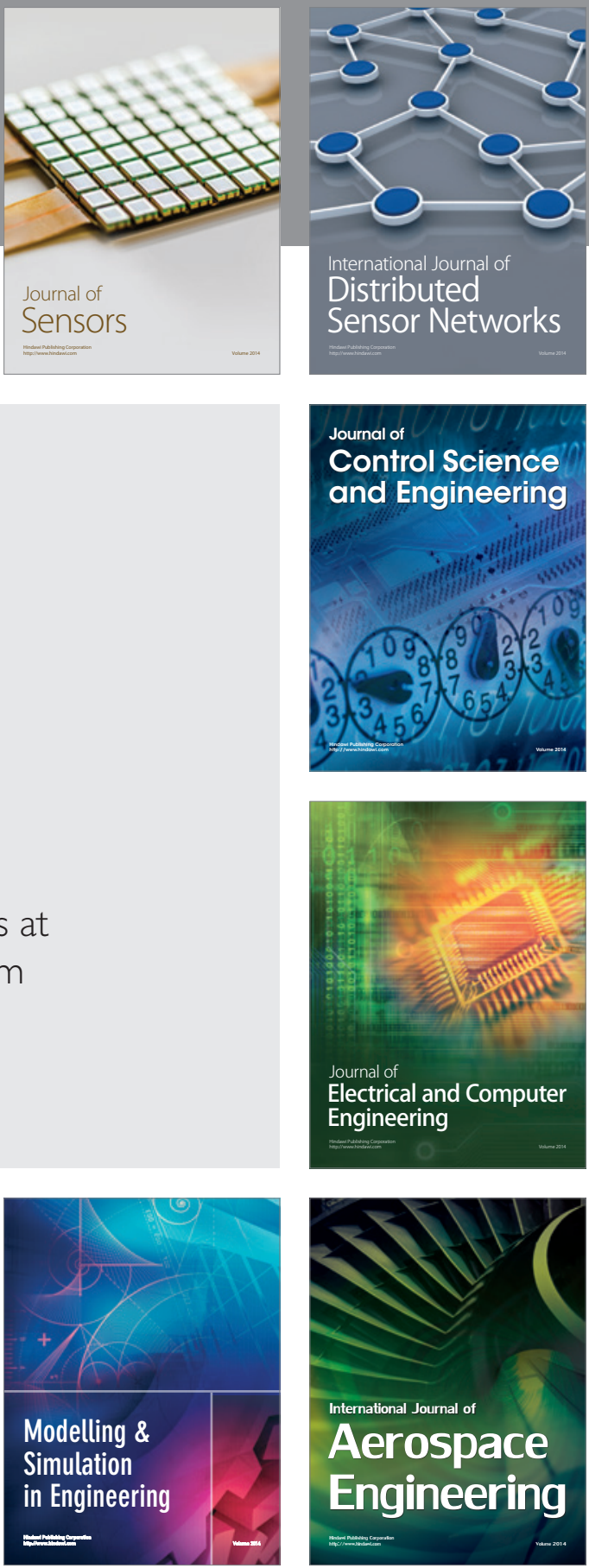

Journal of

Control Science

and Engineering
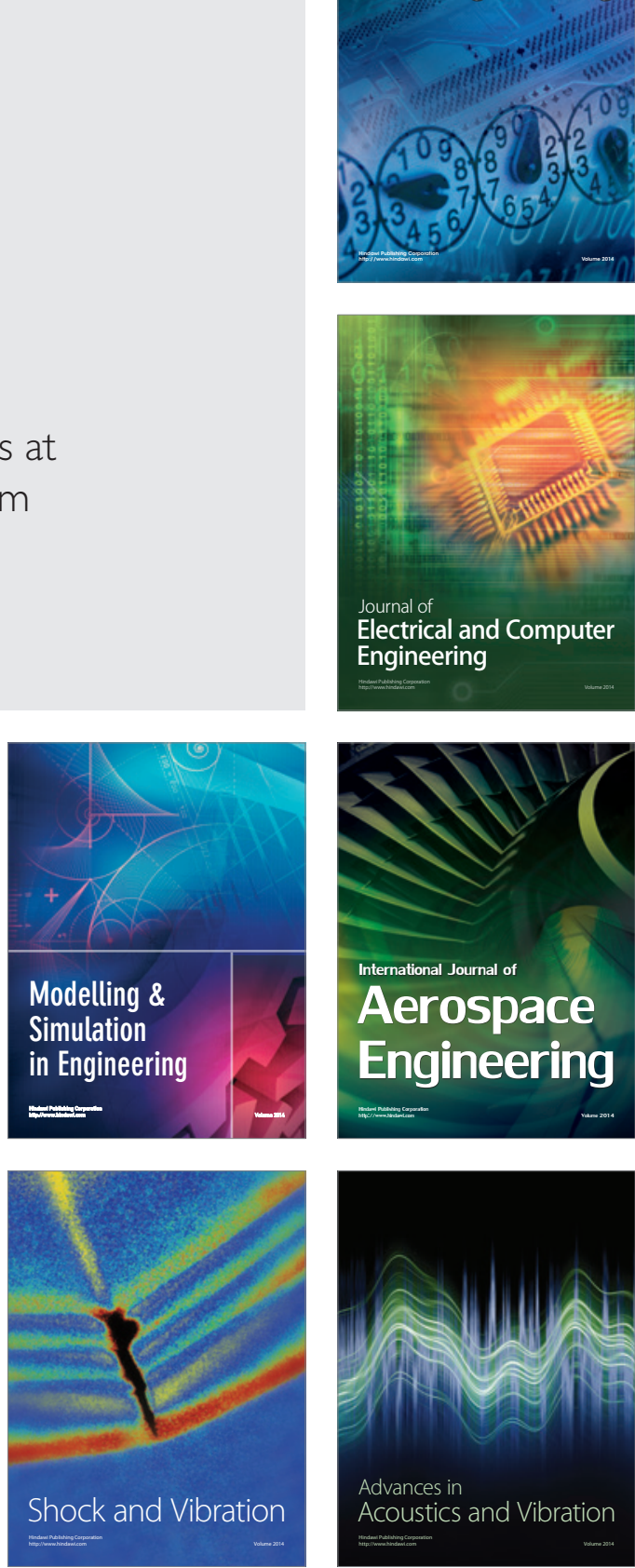